\title{
Brachytherapy as a Treatment for Primary Mucosal Malignant Melanoma of Nasopharynx: A Rare Case Report
}

\author{
Harish Kumar ${ }^{1}$, Arunkumar MN $^{2}$ and Selvaluxmy $\mathrm{G}^{3}$ \\ Assistant professor, Department of Radiation oncology, Cancer Institute (WIA), India \\ Assistant professor, Department of Radiation oncology, Cancer Institute (WIA), India \\ Professor/HOD, Department of Radiation oncology, Cancer Institute (WIA), India
}

Submission: July 13, 2017; Published: July 21, 2017

"Correspondence Address: Harishkumar K, Assistant professor, Department of Radiation oncology, Cancer Institute (WIA), Adyar, Chennai-20, India, Email: hharish_mbbs@yahoo.co.in

\begin{abstract}
Mucosal malignant melanoma (MMM) of the nasopharynx is extremely rare. We report a case of MMM of the nasopharynx in a 35 years old female patient presenting with a polypoidal mass in the nasopharynx. It was increasing gradually and obstructing breathing with occasional bleeding. Computed tomography scan was suggestive of a malignant neoplasm in the nasopharynx. A biopsy of the lesion was done which revealed features suggestive of malignant melanoma with focal melanin pigmentation. Subsequently, endoscopic excision of tumour was done and adjuvant brachytherapy was administered followed by adjuvant chemotherapy
\end{abstract}

Purpose: Primary malignant melanoma of the nasopharynx is infrequently observed. Clinically, patients presents with unilateral epistaxis or nasal block. Mean survival time of 3 - 3.5 years for MMM.

Material and Methods: We have reported the case of malignant melanoma of the nasopharynx. 35 years old female had presented with right side nasal blockage and epistaxis during the six months before diagnosis. Endoscopic resection was performed to completely excise the nasopharynx tumor. Using positron emission tomography/computed tomography examination with 18-fluorodeoxyglucose, the patient was diagnosed with residual nasopharyngeal tumor after surgery.

Results: After repeat endoscopic enbloc resection of leison, intracavitary brachytherapy for nasopharynx was delivered. Patient developed solitary cervical nodal disease occurred 4 months after the Brachytherapy. She underwent Left MRND. After that, external beam radiotherapy was performed on the bilateral entire neck. Thereafter, the patient was given follow up care in the Department of Medical Oncology and planned for chemotherapy until the time of distant progression of the disease.

Conclusion: We have discussed this condition, in lights of its rarity and the need of utilising radiotherapy, despite the mucosal malignant melanoma had been considered as a radio resistant disease, and further more to underline the significance of multidisciplinary approach to deal with treatment of such patients.

Keywords: Brachytherapy; Malignant melanoma; Nasopharynx

Abbreviations: Mucosal Malignant Melanoma (MMM); ENT: Ear-Nose-Throat; CT: Computed Tomography; PNS: Para Nasal Sinuses; PET: Positron Emission Tomography; CTV: Clinical Target Volume; HDR: High Dose Rate; GTV: Gross Tumor Volume; SNMMs: Sino nasal Mucosal Melanomas

\section{Purpose}

Melanomas are tumors emerging from melanocytes which are neuroectodermally determined cells situated in the basal layers of skin, skin adnexas and a portion of the mucosal film. Basic destinations for melanomas are head and neck and the bring down furthest points as they are presented to daylight one of the inclining element. Less ordinarily they happen in the

oral furthermore, genital mucosa, nail beds, conjunctiva, circle, throat, nasal mucosa or nasopharynx, vagina and leptomeninges [1]. Sinonasal threatening melanoma is an amazingly uncommon tumor, with essential mucosal melanoma being more forceful than its cutaneous partner. It represents under $1 \%$ of every western melanoma and $<5 \%$ of all Sinonasal tract neoplasm. 


\section{Cancer Therapy \& Oncology International Journal}

Harmful melanoma in the nasal pit and nasopharynx happens once in a while, from 0.3 to $2 \%$ of every dangerous melanoma, and $4 \%$ of all threatening melanomas of the head and neck [2-4]. Without any characteristic clinical side effects is regularly analyzed in view of histopathological discoveries. The guess is constantly poor, in light of high rates of loco regional repeats or inaccessible metastasis a mid a while or years after determination. The premise of treatment comprises in the surgical extraction. Radiotherapy and chemotherapy have little impact on the expert diagnosis of the infection $[5,6]$. In this paper we have portrayed an instance of threatening melanoma in the nasopharynx and the impact of radiotherapy on the control of this disease.

\section{Material and Methods}

A 35 year old lady had a feeling of nasal obstruction and bleed from the right nostril for approximately 3 times during the period of 6 months. In April 2016, ENT (ear-nose-throat) examination had found leison in nasopharynx. She underwent computed tomography (CT) scan of the paranasal sinuses
(PNS), which was described as an enhancing mucosal lesion in nasopharynx with extension onto bilateral posterior nares more on right side.

In Late April 2016, the patient was admitted to local hospital and underwent endoscopic biopsy of leison. Histological result was mucosal malignant melanoma of nasopharynx.

\{IHC - Positive for S100 / HMB 45, Negative for Pan CK/ CD 45(LCA)\}.

Patient reported to our OPD for further management, Clinical ENT examination revealed residual lesion in nasopharynx. On May 2016, the Positron Emission Tomography/Computed Tomography (PET/CT) examination with 18-fluorodeoxyglucose (18FDG) had been performed, showed metabolically active heterogeneously enhancing soft tissue mass lesion in roof of nasopharynx - SUV 6.0. There were no further distinct pathological changes in PET/CT images in relation to the underlying disease, no signs of lymph nodes or metastatic involvement of other organs (Figure 1).

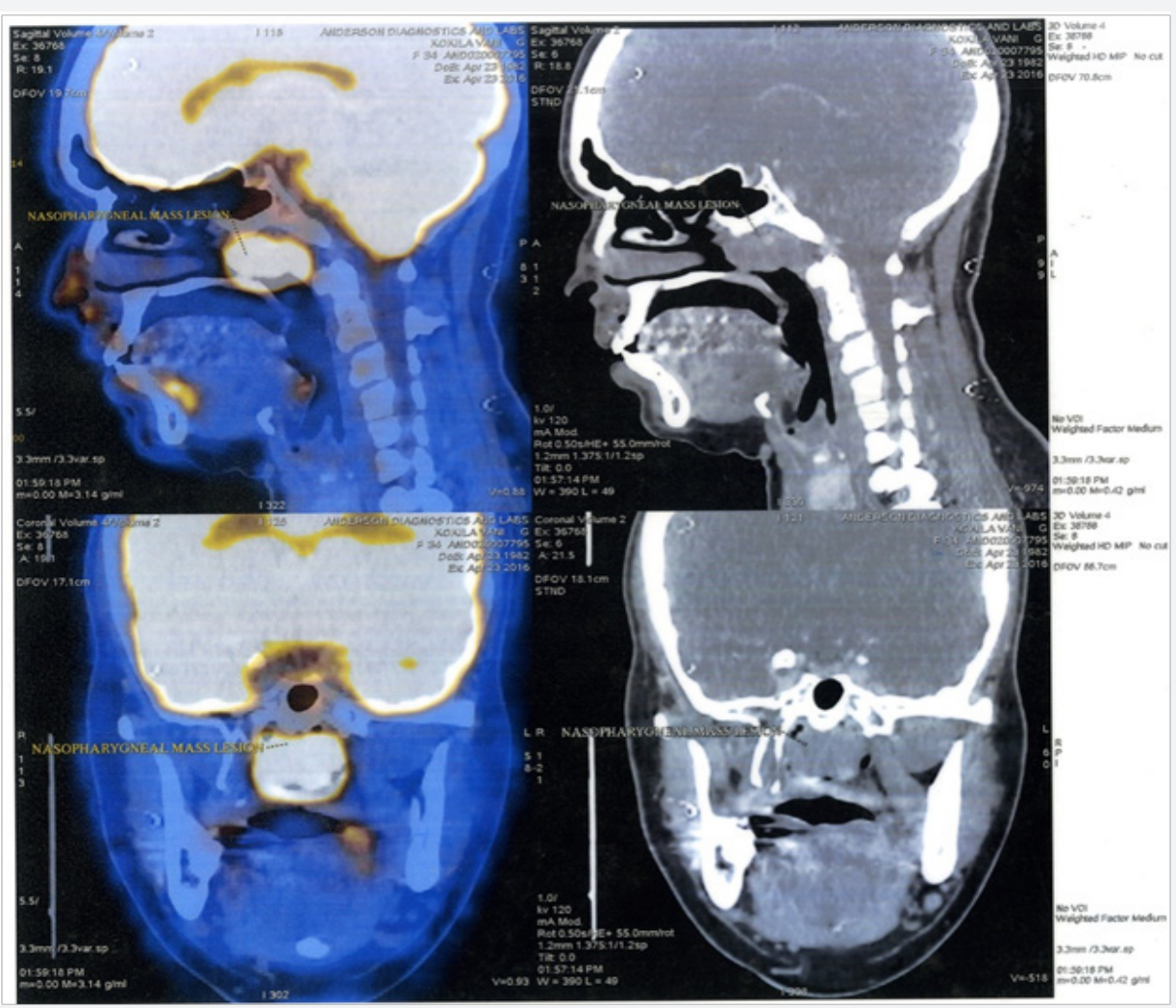

Figure 1: Pre Surgery and pre brachytherapy PETCT Image with residual disease in nasopharynx- Left level II region.

On $14^{\text {th }}$ June 2016, She underwent endoscopic resection under local anaesthesia, post op HPE showed 2x 1.5 x $0.6 \mathrm{~cm}$ residual lesion consistent with mucosal malignant melanoma after IHC co relation (Positve for S100, HMB 45/ Melan A / LCA / Vimentin / CD5/6 and keratin) , All resected margins are free of tumour - pT1Nx. Patient had been presented to multidisplinary board and planned for brachytherapy of the nasopharyngeal cavity. We used a high dose rate (HDR) brachytherapy using
Microselectron HDR® (Nucletron, an Elekta company, Elekta AB, Stockholm, Sweden), with source 192Ir HDR (air-kerma strength was $21698 \mathrm{cGy} \times \mathrm{cm}^{2} / \mathrm{h}$ during the first fraction of brachytherapy), and for nasopharynx using

an inflatable balloon applicator (Figure 2). It is a simple, convenient and secure method that does not require general anesthesia. 


\section{Cancer Therapy \& Oncology International Journal}
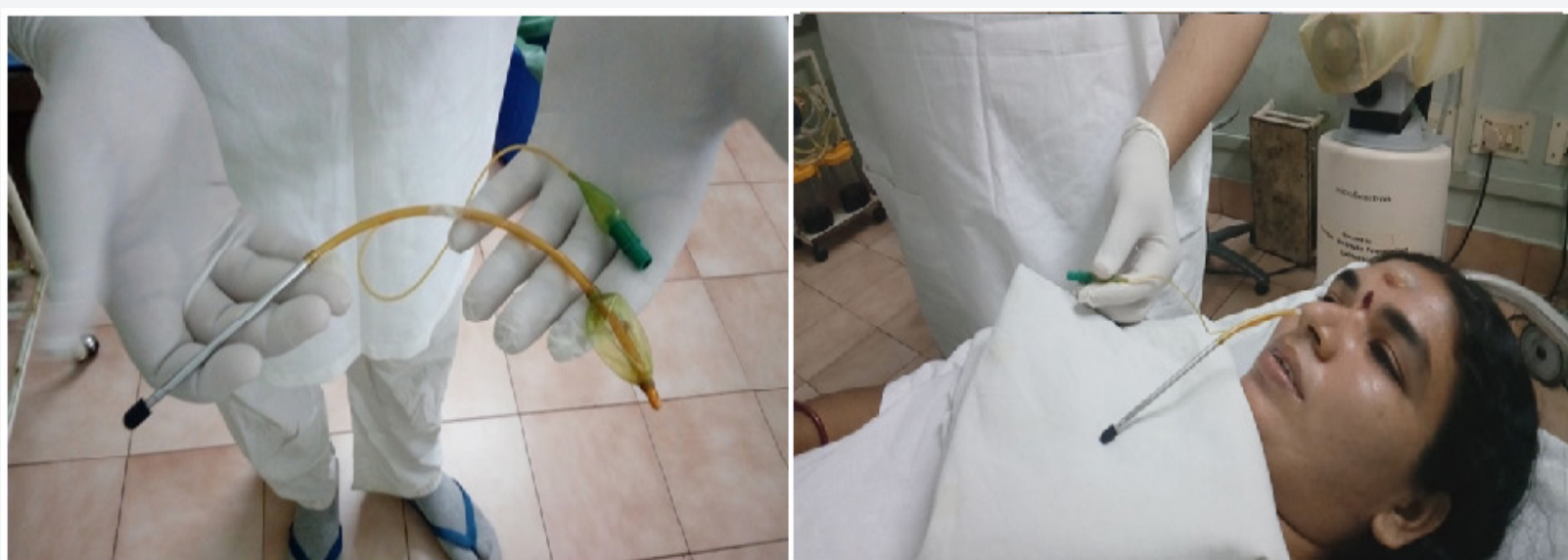

Figure 2: Inflatable balloon applicator - Nasopharynx

The balloon applicator ensures fixation with a good connection between the applicator and the vault of nasopharynx, whilst it reduces the surface radiation dose. The wire with outer diameter $4 \mathrm{~mm}$ had been inserted into the nasopharynx with the deflated balloon which was inflated in the cavity of nasopharynx to the maximal diameter of $30 \mathrm{~mm}$ (Figure 3). Local anesthesia was achieved with lidocaine spray and adrenaline gauze in the nasal cavity. Subsequently, CT images (64 slices) were taken with inserted wire into the closed end of the balloon; the patient was in the supine position.

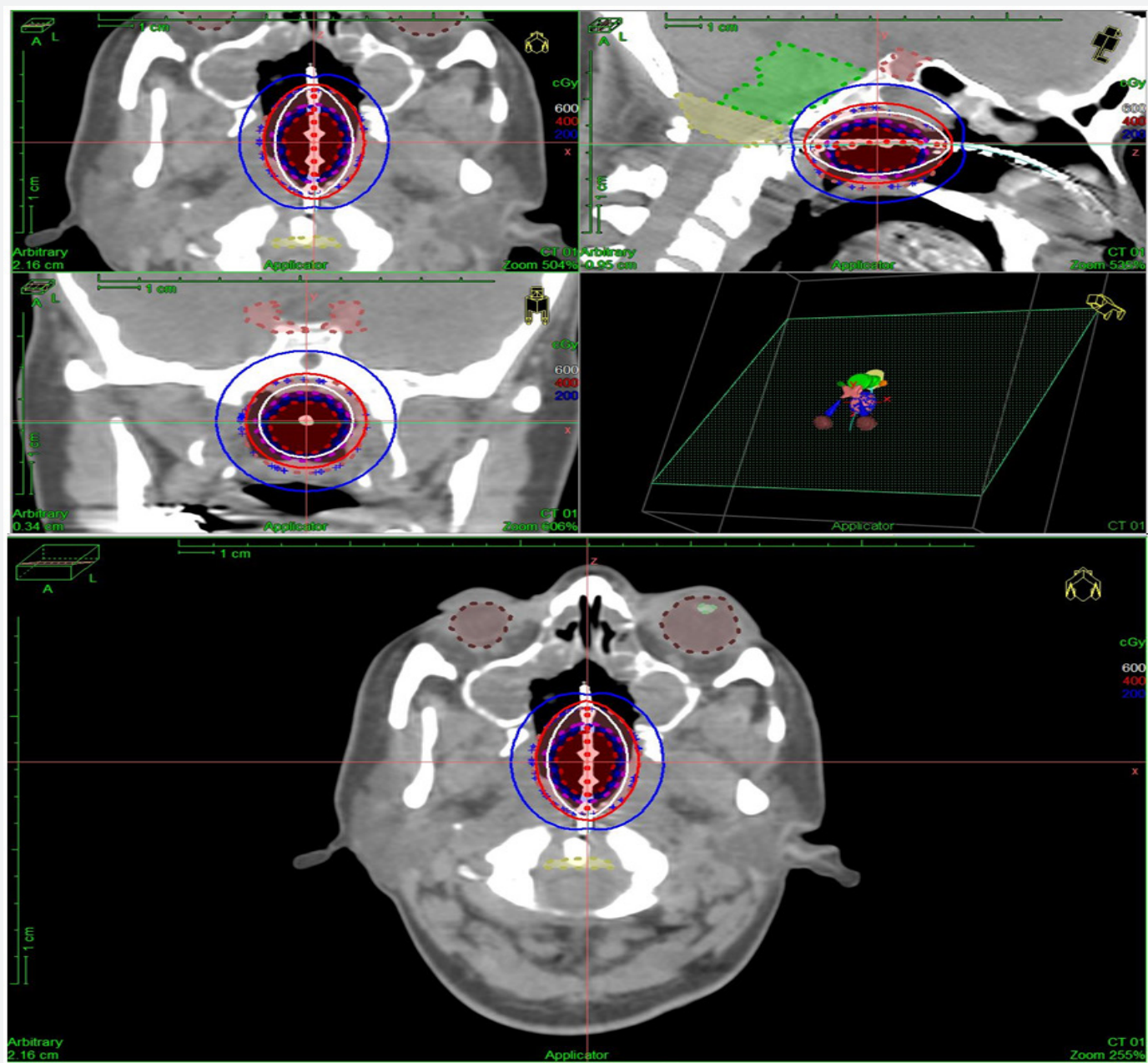

Figure 3: Brachy dose distribution with Isodose curve. 


\section{Cancer Therapy \& Oncology International Journal}

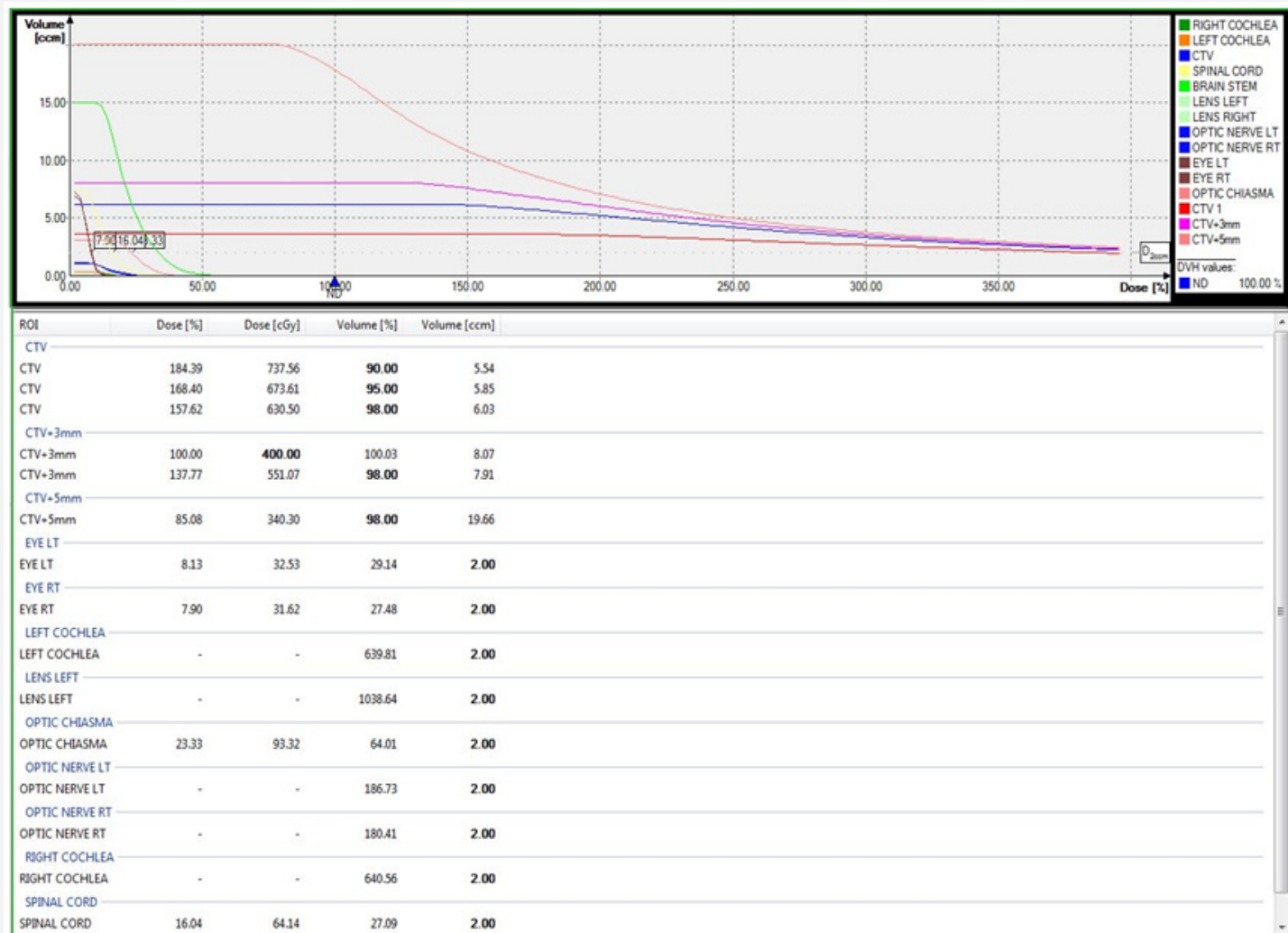

Figure 4: DVH Distribution.

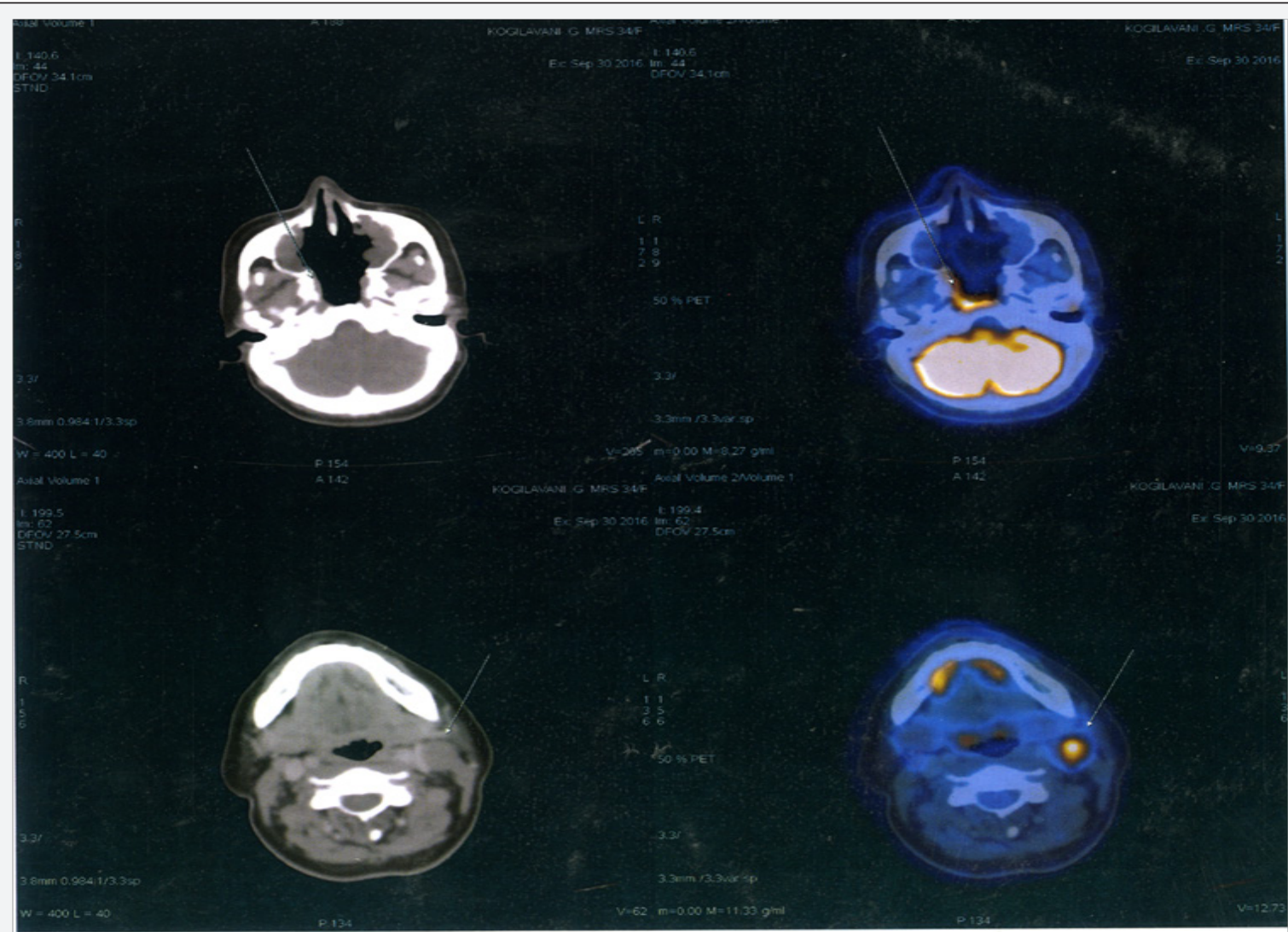

Figure 5: Post brachy PETCT Image with nodal recurrence - Left level II region. 


\section{Cancer Therapy \& Oncology International Journal}

We registered Planning CT images with previous PET/CT images on which has been drawn balloon, clinical target volume (CTV) (CTV was equal planning target volume - PTV) that was equal to balloon plus $3 \mathrm{~mm}$ margins, and surrounding organs (brainstem, chiasma, spinal cord, temporal lobes, the visual apparatus, cochlea on both side) (Figures 4 \& 5). Brachytherapy was planned on the treatment planning system Oncentra $v 4 \AA$ (Nucletron, an Elekta company, Elekta AB, Stockholm, Sweden), number of source positions was 13 , with a spacing of $2.5 \mathrm{~mm}$.

Dose of brachytherapy was 10 x 4 Gy (for $\alpha / \beta$ 0.5Gy EQD2 \{dose in 2 Gy fractions biologically equivalent to dose $\mathrm{D}$ given in fractions of size d, in units of Gray\} was 72 Gy), 2 times per week (Tuesday and Thursday), 100\% dose was $3 \mathrm{~mm}$ from the surface of the applicator, the applicator had diameter $20 \mathrm{~mm}$, irradiated length was $30 \mathrm{~mm}$, D90 (the median dose delivered to 90\%), for gross tumor volume (GTV) was 85\% according PET/ CT. Surrounding organs received a minimum dose of radiation (Figures 2 \& 3).

TD 40 Gy HDR Ir 192 brachytherapy delivered over 5 weeks - 2 days / week from 19.07.16 - 18.08.16 Dose of brachytherapy was high, but malignant melanoma as radio resistant disease requires such high dose. During brachytherapy, dryness and burning sensation in the mucosa of the nasal cavity appeared which was managed conservatively with local lubricants.

\section{Results}

ENT examination after brachytherapy on September 2016 Showed - nasopharynx covered with whitish coating, rest normal, no neck nodes palpable clinically and was kept on regular follow up. In Mid October 2016 - On regular follow up clinical examination revealed Left level II Neck - a palpable solid node, measuring $1.2 \times 1 \mathrm{~cm}$. Needle biopsy (FNAC) had been performed and cytological finding confirmed the presence of metastatic melanoma.

PET CT done on 30 September 2016, showed increased metabolic activity in nasopharynx with no CT demonstrable lesion, mostly recent post RT changes (SUV 9.4) and neck nodes involving left level II - SUV 12.7, no other evidence of distant metastasis (Figure 4). She underwent left MRND On 18th October 2016, post op HPE revealed - LN's 1/31 (Level II - 1/16 with metastatic tumour consistent with perinodal spread and lymphatic tumour emboli, Level III - 0/1, Level IV 0/4, Level V 0/10). Post surgery ENT examination was done which showed Nasopharynx - free, vaulted, without tumour. Neck - without palpable lymph nodes, healed scar.

Thereafter, the patient received external beam radiotherapy to bilateral neck (Level II - V) after multidisplinary board discussion, which was done with a dose of $50 \mathrm{~Gy}, 2.5$ Gy per day (for $\alpha / \beta$ 0.5Gy EQD2 was $58 \mathrm{~Gy}$ ), 3D conformal technique, X-rays energy of $6 \mathrm{MV}$, After radiotherapy was finish, the patient was followed up at the Department of Radiation Oncology. The loco regional finding was without signs of recurrence and referred to medical oncologist for adjuvant maintenance chemotherapy. Case discussed in medical forum and was started on Interferon therapy as adjuvant.

\section{Discussion and Conclusion}

Malignant melanomas are usually found in the skin. But in extraordinary situations they can also arise from mucosal epithelium like nasopharynx and represent $80 \%$ of all sinonasal mucosal melanomas (SNMMs). Another 20\% of lesions are located in areas of the paranasal sinuses, most commonly in the maxillary cavity, followed by the ethmoid, frontal and sphenoid sinuses $[7,8]$. Most patients have non-specific symptoms before the diagnosis. The most common symptoms include obstruction and epistaxis. Pain, diplo - pia and proptosis are very rare. Due to the nonspecificity of symptoms, the majority of lesions are in locally advanced state at the time of diagnosis, since the patient visits their doctor about 6 to 12 months after their appearance [6]. The biggest failure in the treatment of SNMMs is a high incidence of local recurrence. The high local recurrence rates may be a manifestation of the multifocality or clinically unapparent diffuse sub mucosal lymphatic spread of melanoma cells [9]. Distant metastasis has been observed in $51.5 \%$ of patients with a mucosal melanoma of the head and neck [7].

Surgery is the treatment of choice for SNMMs, especially complete resection of the tumor with sufficient free margins. Radiation therapy still holds a controversial role in treating of SNMMs $[10,11]$. Melanoma cells are traditionally considered as radio resistant, because they have a high capacity for repair of sub lethal damages, in particular when we used a conventional fractionation. Therefore, hypo fractionation (higher single dose per fraction) can be considered as a reasonable treatment regimen for this disease $[12,13]$. We decided to do only the local excision of nasopharynx tumour as radical surgical treatment would lead to morbidity as per surgical oncologist opinion based on PET/CT method we decided to radiate the change in the nasopharynx, because sensitivity and specificity of this examination was very high $(98 \%, 94 \%$, respectively) [14]. We have decided for brachytherapy, since the change in nasopharynx was small, and due to the proximity of sensitive surrounding structures. Also, brachytherapy allows the use of high doses of radiation with minimal impact on the surrounding healthy organs.

Our patient had developed regional progression of the disease 4 months after local treatment, but the application of postoperative external beam radiotherapy achieved loco regional control of disease. Nearly one year after diagnosis, no distant disease progression appeared and the patient is alive, and her treatment is continued with chemotherapy (Interferon). We have chosen to discuss this condition, because of its rarity and the possibility of using radiotherapy, although the malignant melanoma was considered as radio resistant disease, and also to emphasize the importance of a multidisciplinary approach to treatment of these patients. It seems that postoperative 


\section{Cancer Therapy \& Oncology International Journal}

radiotherapy may have a role in local control of malignant melanoma, as it had in treatment of our patient.

\section{Reference}

1. Fisher CJ, Egan MK, Smith P, Wicks K, Millis RR, et al. (1997) Histopathology of breast cancer in relation to age. Br J Cancer 75(4): 593596.

2. Schonberg MA, Marcantonio ER, Li D, Silliman RA, Ngo, et al. (2010) Breast cancer among the oldest old: tumor characteristics, treatment choices, and survival. J Clin Oncol 28: 20382045.

3. With lobular carcinoma in the BIG 1-98 trial (2012) In: Thirty-Fifth Annual CTRC-AACR San Antonio Breast Cancer Symposium, 72 San Antonio, Cancer Research p. S1-1.

DOI: $10.19080 / \mathrm{CTOIJ} .2017 .06 .555681$
4. Balducci L, Phillips DM (1998) Breast cancer in older women. Am Fam Physician 58(5): 11631172

5. Chen ST, Lai HW, Tseng HS, Chen LS, Kuo SJ (2011) Correlation of histologic grade with other clinicopathological parameters, intrinsic subtype, and patients' clinical outcome in Taiwanese women. Jpn J Clin Oncol 41(12): 13271335.

6. Metzger O, Giobbie-Hurder A, Mallon E, Viale G, Winer E, et al. (2015) Relative effectiveness of letrozole compared with tamoxifen for patients with Lobular Carcinoma in the BIG 1-98 Trial. J Clin Oncol 33(25): 2772-2779.

\section{Your next submission with Juniper Publishers} will reach you the below assets

- Quality Editorial service

- Swift Peer Review

- Reprints availability

- E-prints Service

- Manuscript Podcast for convenient understanding

- Global attainment for your research

- Manuscript accessibility in different formats

(Pdf, E-pub, Full Text, Audio)

- Unceasing customer service

Track the below URL for one-step submission https://juniperpublishers.com/online-submission.php 